\title{
VISUALIZATION OF VGI DATA THROUGH THE NEW NASA WEB WORLD WIND VIRTUAL GLOBE
}

\author{
M. A. Brovelli, C. E. Kilsedar *, G. Zamboni \\ Department of Civil and Environmental Engineering, Politecnico di Milano, Como Campus, Via Valleggio 11, 22100 Como - \\ (maria.brovelli, candaneylul.kilsedar, giorgio.zamboni)@polimi.it
}

\section{Commission IV, WG IV/5}

KEY WORDS: Data Collection, NASA World Wind, Free and Open Source Software, Volunteered Geographic Information, Virtual Globes, Web, Crowdsourcing

\begin{abstract}
:
GeoWeb 2.0, laying the foundations of Volunteered Geographic Information (VGI) systems, has led to platforms where users can contribute to the geographic knowledge that is open to access. Moreover, as a result of the advancements in 3D visualization, virtual globes able to visualize geographic data even on browsers emerged. However the integration of VGI systems and virtual globes has not been fully realized. The study presented aims to visualize volunteered data in 3D, considering also the ease of use aspects for general public, using Free and Open Source Software (FOSS). The new Application Programming Interface (API) of NASA, Web World Wind, written in JavaScript and based on Web Graphics Library (WebGL) is cross-platform and cross-browser, so that the virtual globe created using this API can be accessible through any WebGL supported browser on different operating systems and devices, as a result not requiring any installation or configuration on the client-side, making the collected data more usable to users, which is not the case with the World Wind for Java as installation and configuration of the Java Virtual Machine (JVM) is required. Furthermore, the data collected through various VGI platforms might be in different formats, stored in a traditional relational database or in a NoSQL database. The project developed aims to visualize and query data collected through Open Data Kit (ODK) platform and a cross-platform application, where data is stored in a relational PostgreSQL and NoSQL CouchDB databases respectively.
\end{abstract}

\section{INTRODUCTION}

Delivering geographic information on Internet started soon after the emergence of World Wide Web in early 1990s with very primitive capabilities (Putz, 1994). However with the emergence of Web 2.0 there has been changes in the way this geographic information is delivered, which is coined by the term GeoWeb 2.0. Web 2.0 is defined as a bi-directional collaboration in which users are able to interact with and provide information to sites and also see the information provided by others, instead of a one-directional communication of Web 1.0, where users merely view the contents of sites (Goodchild, 2007a). GeoWeb 2.0, affected by Web 2.0 being collaborative, is characterized by dynamic 2D maps and virtual globes, being participation-oriented, user-centric, distributed, loosely coupled and rich. On the other hand GeoWeb (or GeoWeb 1.0) is characterized by static 2D map sites, being publishing-oriented, producercentric, centralized, closely coupled and basic (Maguire, 2007).

Another new term, Volunteered Geographic Information (VGI) emerged with the birth of GeoWeb 2.0 and is popularized by Goodchild (2007b). Goodchild argues that humans may act as sensors, build and publish the content themselves, using devices with sensors, for instance mobile devices with GPS receivers. Humans in this scenario may also use their camera to geo-tag multimedia content and Internet connection to publish them. ${ }^{1}$

However, there hasn't been much research in realizing a VGI platform, where the all the contributions from users are visualized in three dimensions. As a result, this study focuses on realizing this kind of platform in the most usable way possible.

\section{BACKGROUND}

Through the transition from GeoWeb to GeoWeb 2.0, the 2D static maps have been replaced by 2D dynamic maps and virtual globes. Virtual globes can be considered not only as $3 \mathrm{D}$, but also as $4 \mathrm{D}$, adding time as the fourth dimension (Brovelli et. al, 2013). Moreover, virtual globes became accessible to developers through using the Application Programming Interfaces (APIs) and as a result to everyone being able to access the Internet, once restricted to only users of highly specialized software tools and often accessible only in scientific environments. There are many virtual globes available to use with different properties. Firstly, some of them requires a specific operating system to run, while others can run on multiple operating systems given that there are different releases for each operating system, or they are platform independent. The platform independent

* Corresponding author 
virtual globes can be considered in two categories, one written in Java, and the other written in JavaScript. Considering the Java based virtual globes require installation of Java Virtual Machine, JavaScript based ones can be regarded even more usable in comparison of platform independent virtual globes, as using a JavaScript based virtual globe, the developed platform is accessible through Web, in other words through all the browsers as long as Web Graphics Library (WebGL) is supported.

Moreover, virtual globes can be distinguished by their license type. Some of them are free and open source, while others are closed, so the source code is not open to the public and released with proprietary licenses. Some of the latter require payment, while others require payment depending on the features provided, or are freeware. As free and open source virtual globes enable easily extend the code to enhance the features, or add/remove features depending on the project requirements, is more suitable for researchoriented projects. In a previous work of (Brovelli et. al, 2013) virtual globes were examined in greater detail.

As a result, the best virtual globe that can be used are the ones written in Java, or better JavaScript and free and open source. In this study NASA Java World Wind, and NASA Web World Wind is chosen to be used to realize the VGI platform, where Java version provides some advanced features that are not yet implemented in Web version, yet the Web version is more usable for the general public.

\section{DEVELOPED SYSTEMS}

In this chapter three applications will be described and compared, where the third application is the one that brings together the strongest aspects of the other two. The first one, PoliCrowd 2.0, is a system that enables collecting, managing and visualizing data in $3 \mathrm{D} / 4 \mathrm{D}$. The volunteered data submitted through mobile devices is visualized on a NASA Java World Wind based virtual globe. However, the system supports mobile devices only with Android operating system and requires installation and configuration of Java Virtual Machine. On the other hand, the second application is crossplatform, which makes data collection possible using simply browsers, or having the application for Android or iOS operating system. Using this application it is also possible to visualize the data in $2 \mathrm{D}$. The third application aims to take these two applications a step further and offer a system where the virtual globe is more usable as its API is for HTML5 and JavaScript, so that the virtual globe is available on WebGL supported browsers, which makes it even more usable than the PoliCrowd 2.0 platform, and displays the data collected through both systems. All three applications are developed using Free and Open Source Software (FOSS).

\subsection{NASA Java World Wind SDK based VGI platform (PoliCrowd 2.0)}

In the PoliCrowd 2.0 system data is submitted through Android mobile devices and georeferenced, using the devices' positioning services, such as GPS. The data is stored in a spatial database and published through standard protocols. Using these protocols, the data is visualized in $3 \mathrm{D} / 4 \mathrm{D}$, and on the virtual globe users are able to manage the data and create customized maps.

In this system Open Data Kit (ODK) suite is exploited for realization of a VGI platform, that allows users to report Points of Interest (POIs), using their Android mobile devices. ODK is composed of three modules: ODK Build, ODK Aggregate and ODK Collect. ODK Build is used to design the survey form that users fill in. It is an HTML5 Web application used to create simple forms, using drag and drop. XLSForm can be used for designing more complex forms. ODK Aggregate is server-side module of the ODK suite, which provides blank forms to ODK Collect, accepts compiled forms from it and stores the data submitted. It can be installed on Google's App Engine or on a local Apache Tomcat server, backed with a MySQL or PostgreSQL database server. Using ODK Aggregate, it is also possible to administer the users. ODK Collect is the client-side module of the ODK suite, where users fill blank forms. Users are asked to provide information about a POI, where its location is registered using GPS, with a possibility of adding multimedia content (image, audio and video). It is also possible to fill a form and send it when the Internet connection is available.

3.1.1 System Architecture: The system architecture of the platform is given in Figure 1. The desktop client is a Java Web Start (JWS) application, based on NASA Java World Wind Software Development Kit (SDK), which visualizes the data collected through the mobile client in 3D/4D. The mobile client is an Android application, ODK Collect, through which the data is sent to ODK Aggregate server. The desktop client is able to connect to and retrieve data from any WMS and ODK server, including the ODK server of the project. PoliCrowd server is deployed on the GlassFish Java 2 Enterprise Edition (J2EE) application server backed with a PostgreSQL relational database to store all the data related to the platform, such as the users' credentials, the list of servers that have been used by the users, the list of projects that have been created by the users, along with the projects themselves (the added layers and their on/off status, the state of the globe - the location of the point of view and camera orientation), and the data uploaded by the users as an update of the POIs displayed on the virtual globe.

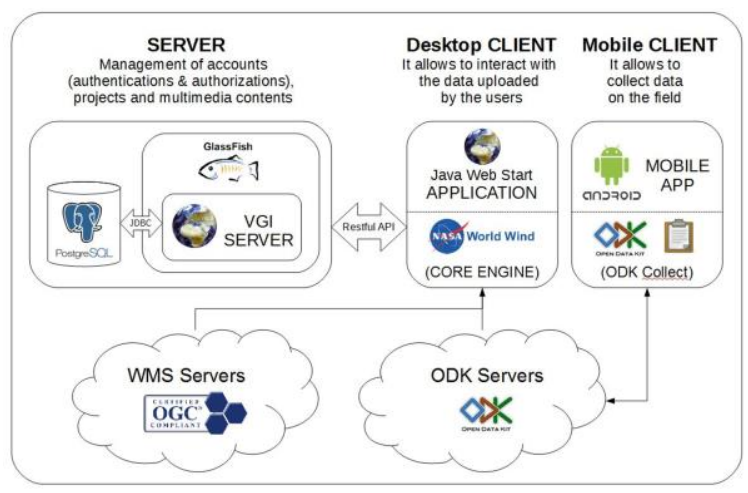

Figure 1. System architecture of PoliCrowd 2.0 
3.1.2 Functionalities: After the first PoliCrowd, focused on the cultural and tourism heritage of the Como city in Northern Italy (Brovelli et al., 2014), PoliCrowd 2.0 is developed, which is entirely general-purpose. PoliCrowd 2.0 platform allows users to create a new project that suits to the specific topic of interest (environmental, social, educational, cultural, scientific, etc.), and save the project in a catalogue available for the whole community, by enabling users to connect any WMS and ODK server and adding the layers that are available and wished to be included in the project. The first level of information consists of WMS layers, which can be base layers, such as OpenStreetMap (OSM), the aerial orthophotos and satellite imagery, thematic maps made available through geoportals of institutional administrations, such as INSPIRE and national geoportals, or any other WMS compliant server. The second level of information is the crowdsourced data stored in ODK Aggregate servers, collected through ODK Collect mobile client.

The related data is displayed in a window once the marker is clicked, as shown in Figure 2. This data can be enriched by new data (comments or multimedia content, such as images, audio and video) by any authenticated PoliCrowd user. Furthermore, a separate web application, based on Java Server Faces (JSF) is developed to remotely administer the platform contents, using CRUD (create, read, update, delete) operations on the database.

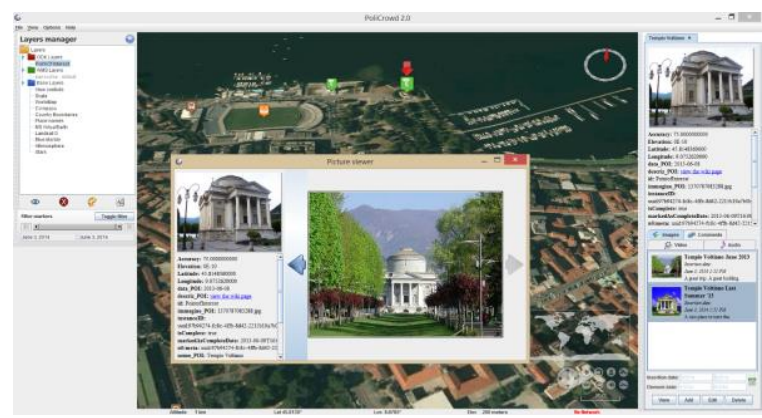

Figure 2. The visualization of data for the clicked marker

Unlike WMS layers, where the style of them are defined on the server side, the platform allows customization of the ODK layers' icons. Users can select an attribute among all the attributes of the ODK layer to be used to set the icons. Firstly, for the selected attribute, icons are assigned automatically. Later, user can change the individual icons, using the collection already available, or different icons can be uploaded from the local environment.

Moreover, a temporal slider is available to filter both the ODK layers and their multimedia content, introducing the 4th dimension in data visualization. While the ODK markers are filtered according to the date they were submitted through ODK Collect, the multimedia can be filtered by the date they were created or they were uploaded to the platform. The date multimedia content is created and its description can be added by the users as metadata while uploading them to the platform.

While any user can access and view all the projects, only the project owner has the privilege to edit his/her own project, which means the project's metadata (project name, project description, etc.), the layers used and the marker styles can be edited only by the authenticated user. Moreover, a new project can be created only by the authenticated users.

\subsection{Cross-platform VGI application}

As ODK suite allows to realize a VGI system, that enables data collection only using mobile devices with Android operating system, a new cross-platform application has been developed (http://viaregina3.como.polimi.it/app/). This application, being cross-platform, is available on desktop accessed through browsers and on mobile devices, both on browsers and as an application. On desktop, the application is tested on Linux, Windows and OS X operating systems, on browsers Google Chrome, Mozilla Firefox and in case of OS X also on Safari. On mobile, the application is available for Android and iOS, and on these operating systems it is tested on browsers Google Chrome, Mozilla Firefox and in case of iOS also on Safari. However, although it is not tested the application is supposed to work on all modern browsers and can be compiled as an application for also Blackberry 10, OS X, Ubuntu, Windows, Windows Phone 8 (WP8), LG WebOS and FireOS.

The application is developed within the framework of Via Regina project (http://www.viaregina.eu), which is an INTERREG project (Crossborder Cooperation Operational Programme Italy - Switzerland 2007-2013) developed thanks to the cooperation between Switzerland and Italy. The project aims at promoting, rediscovering and enhancing the naturalistic, artistic and cultural heritage along Via Regina, overlooking the West coast of Lake Como in the northernmost part of Italy (Antonovic et. al, 2015).

The application is primarily developed to collect data, suitable for the scope of Via Regina project. However, the application can easily be adapted for a different or a more general-purpose context.

3.2.1 System Architecture: The system architecture of the cross-platform application can be seen in Figure 3. The application is developed using Apache Cordova, which is a mobile application development framework. Cordova enables to use one code base to target multiple platforms, easing the process of having an application that can run cross-platform. The map is built using mobile-friendly Leaflet library. The data is stored in document-oriented NoSQL CouchDB database that stores the data in JavaScript Object Notation (JSON) format. PouchDB is integrated into the system, since it enables applications to store data locally while offline and synchronize it with CouchDB when the application is back online. Besides the data stored in PouchDB/CouchDB, also the data in ODK Aggregate server of Via Regina project can be displayed. The ODK Aggregate server of Via Regina project is backed with PostgreSQL database, extended with PostGIS, so that the data is available through GeoServer, published with Open Geospatial Consortium (OGC) standards. In the application, JSON with padding (JSONP) format is used to overcome the crossdomain restrictions. 


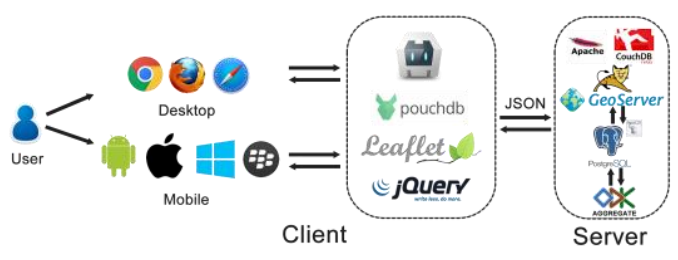

Figure 3. System architecture of the application

3.2.1 Functionalities: Firstly, users are asked to register giving information on their gender, age and work status only for statistical purposes. After registration, users can start using the application. Most importantly, the application lets the user to insert a POI, which has attributes relevant to the context of the Via Regina project. The steps to insert the POI is given in Figure 4. Mainly, the categorization of the POI, its rating, comment (optional), picture (optional) either uploaded from local environment or taken using the camera of the mobile device (only in case of application) is submitted. The marker is automatically placed in the user's position using the device's location services (Wi-Fi, Bluetooth, cellular networks or GPS) in case they are available and user gives consent.

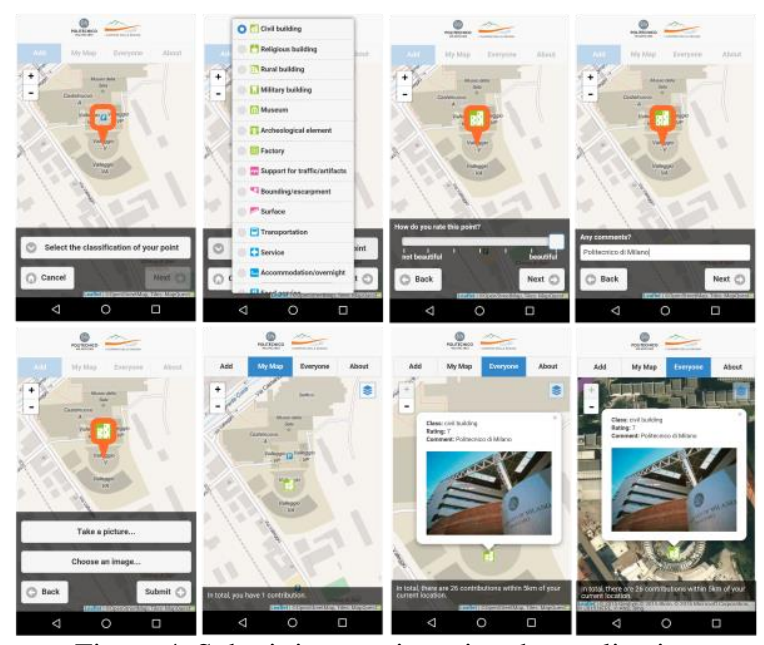

Figure 4. Submitting a point using the application

The inserted POI is then placed on the map as an icon, which opens a popup upon clicking showing inside the data inserted.

Moreover, it is possible to see the points inserted by the user, and by everyone. In both cases the POIs are aggregated based on their category and displayed on the map using the color dedicated to each category. It is also possible to switch the basemap between OSM and Bing Aerial imagery and see what the categories are in a legend. Both can be seen in Figure 5.

Additionally, the language is automatically switched between English and Italian, based on the language of the browser (in case of browser) or the operating system (in case of application).
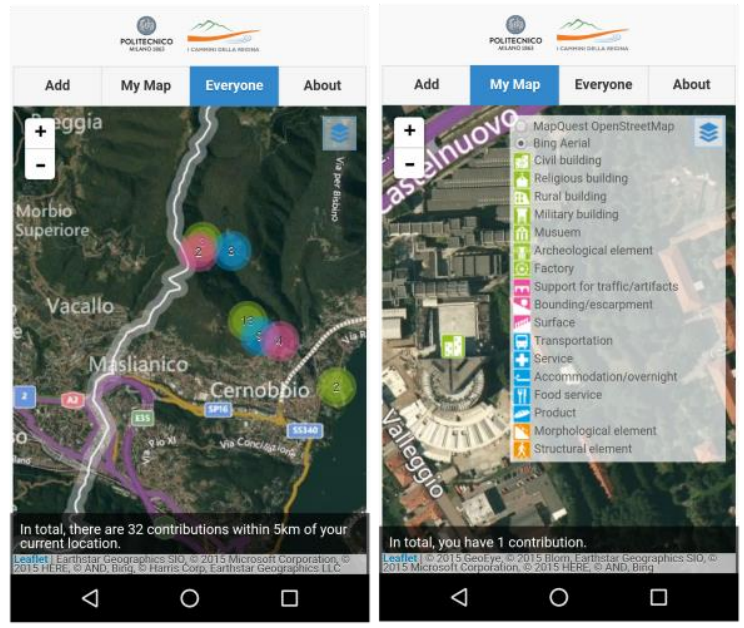

Figure 5. All POIs aggregated based on their category (left) and the legend (right)

\subsection{NASA Web World Wind API based VGI platform}

The virtual globe, developed using NASA Web World Wind API (http://viaregina3.como.polimi.it/WorldWind/) is able to display data stored in the ODK Aggregate server of Via Regina project and in CouchDB database of aforementioned cross-platform application, both on desktop and on mobile device browsers, as long as WebGL is supported. It is also responsive as Bootstrap is used.

The architecture of the platform is shown in Figure 6.

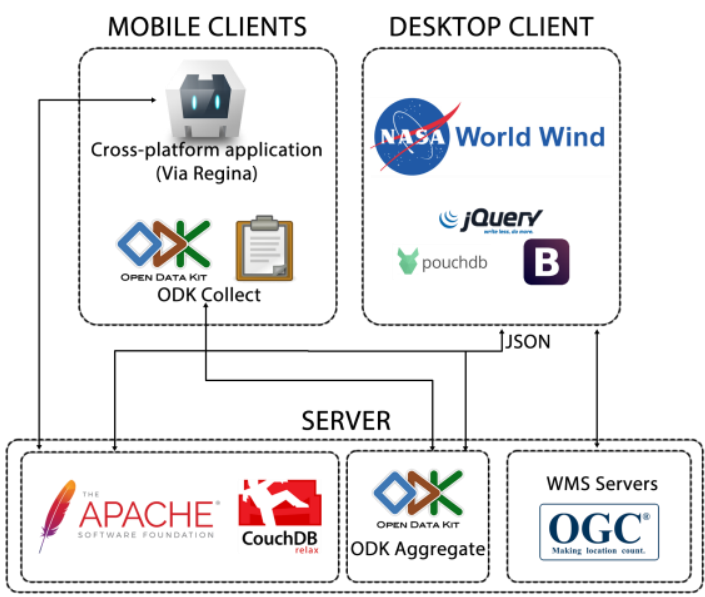

Figure 6. System architecture of the platform

The general look of the virtual globe is given in Figure 7, where yellow markers represent the ODK POIs and red ones represent the CouchDB POIs. The area of the Via Regina project's scope is highlighted with red borders. 


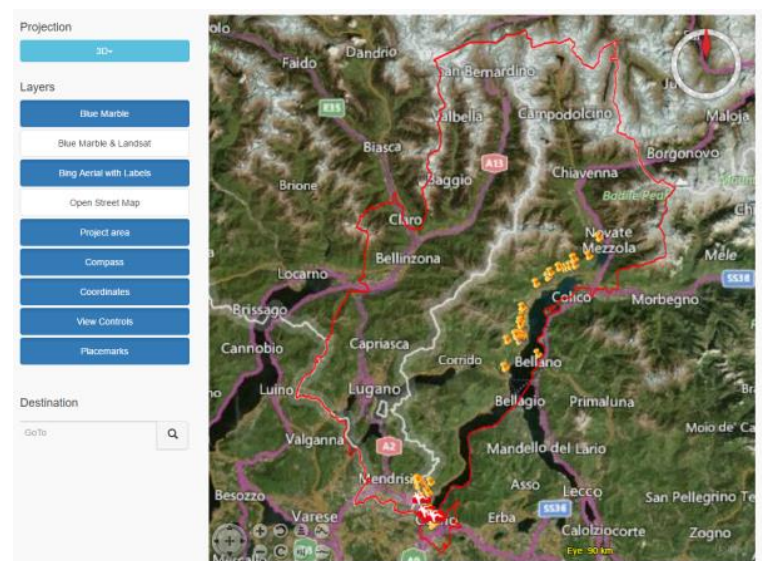

Figure 7. ODK POIs and CouchDB POIs on virtual globe, based on NASA Web World Wind API

The data of each POI is displayed inside a popup upon clicking the marker as shown in Figure 8.

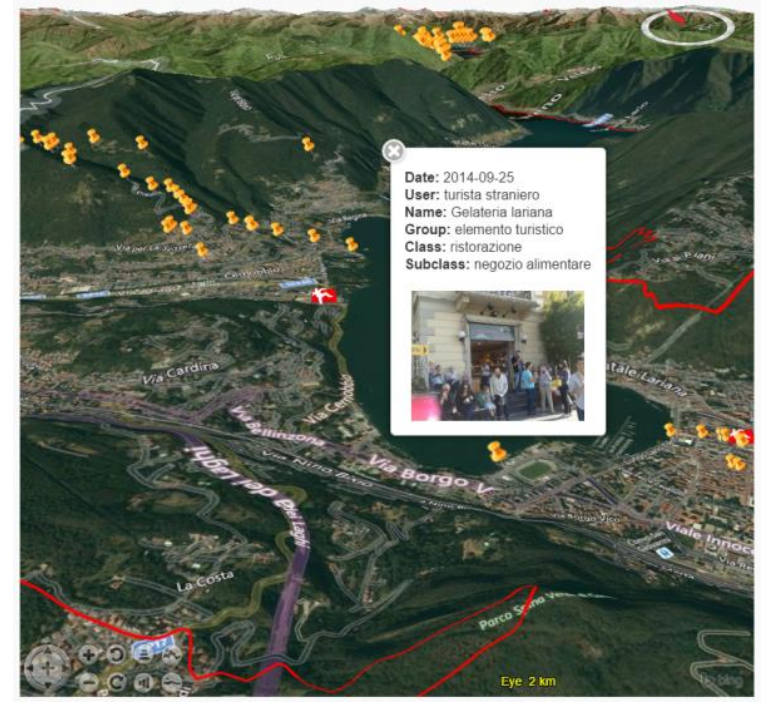

Figure 8. Popup window of a POI

Moreover, the NASA Web World Wind API offers some base maps, such as Landsat imagery, Bing aerial imagery and OSM. It also has view controls and controls for compass, coordinates and geocoding, where all of them can be seen in Figure 7.

\section{CONCLUSIONS}

Advancements in computer graphics, Web 2.0, and eventually GeoWeb 2.0 have brought virtual globes even on Web and VGI systems into existence. However there has not been much research in integrating virtual globes with VGI systems. The study focuses on this integration, placing importance on the usability of the platform, using FOSS. As a result, NASA Web World Wind API is used to realize the virtual globe, displaying crowdsourced data collected using different applications, where both the virtual globe and the applications used (except ODK Collect) to collect the data are cross-platform. The NASA Web World Wind API based platform can be considered as a continuation of the previous platform, PoliCrowd 2.0, to take it a step further, by basing it on Web standards, so that it is accessible through WebGL supported browsers on different operating systems and devices.

Future work should be able to connect to the PoliCrowd server, as currently the platform can merely connect to the ODK Aggregate server and the CouchDB server. This feature is necessary to integrate the crowdsourced data wholly into the application. Future work should also add the $4^{\text {th }}$ dimension to filter both the ODK Aggregate and the CouchDB POIs by time.

\section{REFERENCES}

Antonovic M., Brovelli M.A., Cannata M., Cardoso M., Kilsedar C.E., Minghini M., Zamboni G. (2015). Promoting slow tourism through FOSS4G Web mapping: an ItalianSwiss case study. Geomatics Workbooks $\mathrm{n}^{\circ} 12$ - Proceedings of FOSS4G Europe Como 2015, pp. 99-104.

Brovelli M.A., Hogan P., Minghini M., Zamboni G. (2013). The power of virtual globes for valorising cultural heritage and enabling sustainable tourism: NASA World Wind applications. International Archives of the Photogrammetry, Remote Sensing and Spatial Information Sciences, Volume XL-4/W2, pp. 15-120.

Brovelli, M. A., Minghini, M. and Zamboni, G. (2014). Three dimensional volunteered geographic information: a prototype of a social virtual globe. International Journal of 3-D Information Modeling 3(2), pp. 19-34.

Goodchild, M. F. (2007a). Citizens as voluntary sensors: spatial data infrastructure in the world of Web 2.0. International Journal of Spatial Data Infrastructures Research 2, pp. 24-32.

Goodchild, M. F. (2007b). Citizens as sensors: the world of volunteered geography. GeoJournal, 69(4), pp. 211-221.

Maguire, D. J. (2007). GeoWeb 2.0 and volunteered GI. Proceedings of the Workshop on Volunteered Geographic Information, University of California, Santa Barbara, CA, pp. 104-106.

Putz, S. (1994). Interactive information services using World-Wide Web hypertext. Computer Networks and ISDN Systems 27(2), pp. 273-280. 